\title{
The Principles of Building Harmonious Moral Education Target in University
}

\author{
Yanjun Dai \\ School of Marxism \\ Dalian University of Technology \\ Dalian, China \\ e-mil:daijy@dlut.edu.cn
}

\author{
Hua $\mathrm{Wu}$ \\ School of Marxism \\ Dalian University of Technology \\ Dalian, China \\ e-mail:xiaolaba3330@163.com
}

\begin{abstract}
A harmonious moral education target is the value orientation for the system of harmonious moral education and it plays an important role in building a system of harmonious moral education in the university. Therefore we should adhere to the people-centered principle, grasp the principles of scientific nature, and strive to the orientation, advance with the times and the innovative development of the principles to realize the grand goal of building the harmonious moral education target. At the same time, we should also pay attention to the standardization, pertinence and operability in building the target of a harmonious moral education.
\end{abstract}

Keywords-Harmonious moral education target; Building; Principles

\section{INTRODUCTION}

Harmonious moral education is a pattern what is "starting with the students needs and the need of society, following the law which is benefit to people's body and mind development, regulating the relationship between many elements of moral education." [1]This model can promote the students get comprehensive and harmonious development. Harmonious moral education system is embodied in the various elements of moral education. Harmonious moral education target is one of the elements. It is an unification of moral education target in the same period and a harmonious and strong connection among different periods. And it should be in harmony and unity to avoid conflict and confrontation between the individuals and groups. A harmonious moral education target is the value orientation for the system of harmonious moral education and it plays an important role in building a system of harmonious moral education in the university. Therefore, we should adhere to the people-centered principle, grasp the principles of scientific nature, and strive to the orientation, advance with the times and the innovative development of the principles to realize the grand goal of building the harmonious moral education target. Only in this way, we can build and optimize the harmonious moral education target. We also can make the moral education effectiveness and pertinence.

\section{GRASP THE PEOPLE-CENTERED PRINCIPLE}

If we want to build a harmonious moral education target, we should adhere to the people-centered principle. It means that teachers need to correctly balance the relationship between teachers and students to give full play to the initiative of students, develop their creative thinking in moral education. It can promote students' free, comprehensive and harmonious development.

\section{A. Pay Attention to Students' Individual Character Development}

First of all, we should pay close attention to students' individual character development and be approached in a comprehensive, objective and rational manner analysis about the dominant position of students in the process of moral education activities. In addition, the moral education target should adapt and meet the needs of the students' free development. The exposition of "human nature" in On the Outline of Feuerbach has long been a definition. Marx believes that "human nature" is the sum of all social relations. In Marxism on the theory concerning man's development, Marx specially emphasized on the man's freely completely development, emphasized on individual liberation or individual realizing. In short, Moral education should meet the needs of the student all-round development of morality, intelligence and physique. So the needs of the human comprehensive development are very important criteria to build moral education target. In a word, we must adhere to the peoplecentered principle for building a harmonious moral education target. This principle is one of the most fundamental; it reflects the nature of moral education.

\section{B. Promote the Sustainable Development}

Secondly, we should promote the sustainable development of the moral education target. Moral education target must maintain sustainable development. We hope that the target can help the college students to deal with the man and nature, man and society and people and their relations correctly in learning and life. College students achieve harmonious idea and belief by adjusting these relationships; it can make them engaged in the construction of harmonious society. In practice, the students can learn how to promote the coordinated development of individual. 


\section{Be Focus on The Students' Comprehensive Quality.}

Thirdly, we should focus on the students' comprehensive quality. Albert Einstein who is the most famous scientist in the world once wrote in "On Education": Schools should have a target, which is when a student leaves school; he is a harmonious people, rather than a specialist.[2] Cultivating compound talents is an inevitable requirement of social needs and the development of higher education. A complex talent who has the innovational ability and the knowledge of science and technology is required in a changing world. Moral education is an important task, related to the overall quality of higher education personnel, life and values to develop; it is an important part of the personnel of higher education. Harmonious moral education as a good mode of moral education should deliberate the development of students' comprehensive quality. We can under the guidance of the comprehensive target system to training a lot of students who have the professional knowledge, comprehensive and harmonious moral quality and physical and mental quality.

\section{GRASP SCIENTIFIC PRINCIPLE}

To build a harmonious moral education target should insist on the scientific principle. We should use the scientific methods; grasp the regularity, systematicness and gradation of moral education target. This target is not only in line with the party's policies and the related theory, also accord with college students' ideological characteristics. It can be accepted by students happily.

\section{A. Hold Three Laws for Building Moral Education Target}

Firstly, we must hold by three laws to build moral education target. The three laws are rule of people's moral character formation and development, rule of education and self education and rule of moral knowledge and practice unity.

- The rule of people's moral character formation and development is the fundamental bases of moral education activities in university. We should take the ideological and moral state of college students who live in current society to build the moral education target. Marx wrote "Thought process what is in people's mind is determined by the people's material life conditions". [3] That means the root of people's moral character development exists in the life process. At first, we must evaluate college students' ideological and moral level correctly, then we can set moral education target. This target should be appropriate beyond present college students' ideological and moral levels and has promoted the possibility of ideological and moral level. But this transcendence is limited; it is not too high to reach with endeavor for students.

- The rule of education and self education is another important basis for making a successful moral education target. Teachers and students are basic elements in moral education activities. The rule of education and self education reflects the interaction between the teacher and the student. This rule therefore attempts to suggest: in moral education, teacher and student's role be reshaped and teacher-student relationship be best readjusted. So we must follow this rule to build the moral education target. Building the moral education target system should pay attention to the realization of the college students' self education. Especially we should pay attention to the formulation of the moral education target content. The contents of moral education target include ideological quality, moral quality and so on. The target of moral education is to transform these contents as an organic part of students' self consciousness. At the same time, we should use all kinds of methods to arouse the initiative of educators in the system of moral education target methods implementation. Educators should play a leading role to guide students for completing the self education, so students can realize the internalization of moral education.

- The rule of moral knowledge and practice unity emphatically solves the externalization of undergraduate moral education problems. The achievement of moral education target is measured by the results what is good or bad behavior habits through the practice of moral education. Therefore, moral education target standard of cognition, emotion, will and other elements in cultivation must fulfill ultimately the college students' good behavior habits. So we should follow the rule of moral knowledge and practice unity to build moral education target, combine with reconstruction of the subjective world and objective world, and be emphasis on the moral education practice process.

\section{B. Grasp the Hierarchy of Building Target}

Secondly, we must grasp the hierarchy of building. Moral education target in university has complete structure. It is a target network which is "a horizontal interpenetration, vertical stratification, internal orderly combination of target group"[4]. Grasping the principle of hierarchy has contributed to get achievement of moral education practice. It is also conducive to evaluate effects of moral education practice. Therefore, building of the university moral education target must be based on the physical and mental characteristics and the actual situation of college students, follow its moral character formation and development regularity and the characteristics of the university education. If we do like this, each target for the moral education, but it is also a progressive layer of process. The purpose is to achieve the harmonious development of each target of moral education.

The moral education target in university consists of two parts: one is the basic factors layers, the other is development layers. The basic factors mainly include seven elements; they are mental health, humanities accomplishment, legal discipline and morality, ideal and belief, behavior habits. These seven elements are increased position in turn in moral education target. Completion of each target is developing previous target, and become foundation of next target at the same time. 
The development layers include three layers: short-term goal, medium-term goal, long-term goal. The short-time goal of harmonious moral education reflects the needs of the development of today's society. It is an expectation about the results of moral education. It has the characteristics of the reality and concreteness. The medium-term goal is a target which is the moral education workers can achieve after a long time of hard efforts, and it is also a feasibility of moral education outlook. It is an excessive goal of long-term goals and short-term goals. The long-term goal of moral education paints the blueprint of harmonious moral education. It has a strong guiding significance about short-term goal and mediumterm goal. If we want of reach the long-term, we should achieve the short-term goals and medium -term goals one by one. The development layers of harmonious moral education guide the practice of university moral education.

\section{Grasp the Systematicness of Building.}

Thirdly, we must grasp the systematicness of building. This systematicness refers to the phased goal of moral education. The ultimate goal of harmonious moral education is to nurture students to be builders of socialism and practitioners of the socialist core value system. This ultimate goal is not achieved overnight. Its implementation depends on the realization of targeted isolation. Therefore, we must grasp the stage of moral education. At first, we should pay attention to build different target in different stages of college students' development. For example, some scholars give advice about moral education target, just like "Growth-Adults-To be a useful person" [5] and so on. These pieces of advice give expression to the periodicity of moral education. Then, we should also pay attention to the contents and forms of moral education in different stages. The aim is to reduce students' resistance in the moral education work, enhance their interest in participating in the moral education practice.

\section{GRASP ORIENTED PRINCIPLE}

Harmonious moral education target not only indicates the political direction for the whole process of moral education activities, restricts the moral education activities in university of the system establishment and implementation of other elements, also affects the harmonious operation of the moral education system in university, more directly affects the effect of moral education, so harmonious moral education target in university should be guided by the correct guidance.

Firstly, we should grasp the orientation of ideal and faith. The content of the moral education target is rich and colorful. But the status and roles of every target is not parallel and same. In the process of the construction of the moral education target, political goals should be throughout the course of the moral education, occupy the dominant position, dominate the other goal of moral education, and determine the nature and direction of harmonious moral education target in universities. So basically, all the ultimate goals of moral education are to realize the political aim of the certain social classes or groups. The orientation of political goal makes it obvious which are the characteristics of moral education. And ideal and faith target is core target of the political goals of harmonious moral education in university, so if we grasp the guidance of ideal and faith goal, we also adhere to the directionality of the political goal.

Secondly, we should work out schemes for moral education activities and complete moral education practice and the practice of moral education effect evaluation and feedback under the guidance of harmonious moral education target. Harmonious moral education target in university not only indicates the direction for the whole process of moral education activities, also affects the content of moral education in colleges and universities, construction of the consultation, or even directly affects the realization of moral education in university. In addition to building of harmonious moral education goals in university, we should give prominence to the moral education in the core position of political goals, adhere to the correct political orientation, embody the essence of moral education, highlight the theme of moral education, drive the other side of the moral education target standards implementation when we committee to the construction of moral education in university.

\section{GRASP INNOVATIVENESS PRINCIPLE}

Establish a brand-new, the reality of the moral education target, we can rapidly changing the face of the world, and to adapt to the changing its own development. We should constantly innovate moral education goal, it should reflect the times. Therefore, we must grasp innovativeness principle to build the moral education target in university.

We should continuously develop the content of the moral education target. The contents of the moral education target are different in different times. In the feudal society, Confucian thought moral education is the all education. It emphasizes that the "benevolence" is the core of education, the aim of education is to cultivate "beneficent humanity". In modern China, moral education has become an important part of education. The content of the moral education target has turned into "Educating Five Domains Simultaneously", the aim of moral education is to cultivate "free and beautiful personality". In the beginning of the P.R.C, comrade Mao Zedong pointed out that the teachers should attach great importance to the ideological and political quality of man, the aim of moral education is to cultivate the talents with ability and political integrity. In the beginning of reform and open, comrade Deng Xiaoping assessed the situation, he asked to formulate new moral education target, the aim of the moral education is to develop students' good morality and habits, making them become the "new persons with four haves". Therefore, along with the development of times, the moral education target has developed. As mentioned before, those moral education target is not suitable for today's social development demands of college students' moral development, so we must build a new and harmonious moral education target in university, this target should reflect the age characteristics and special moral education of university in the socialist market economy system of China. The harmonious moral education target in university not only include the 
traditional "morality, intelligence and physique", and should include the college students' will, behavior, emotions, etc.

Each era has its own spirit; innovation has become "the soul of national prosperity, and personal qualities of the core driving force for the development of the country"[6] in today's rapidly developing age. The constant change of modern society requires that the content of moral education target must innovate, and adapt to the needs of social development. Innovation is driving force what makes the moral education target and the social developments are more harmonious. So building of harmonious moral education target must be based on the reality and face to the future. We should be absorbed and drawn upon fruits of human civilization, be realization of Chinese and western culture from each other and complement each other, to constantly enrich and innovate the contents of moral education target. Our traditional culture should also be given in the era of new, innovative development.

\section{Pay AtTention to Some Questions in Building}

When we build the harmonious moral education target in university, besides these four principles, we should also pay attention to some questions in building.

Firstly, we emphasize the standardization of the construction. Standardization is inseparable from the request. Our idea of building should be rooted in "deep understanding of higher education law, law of socialist market economy, college students' physical and mental growth pattern"[7],be rooted in deep mastery of the contemporary excellent achievement of human culture and the quintessence of Chinese traditional culture. Only by following these two specifications, we can build the more harmonious moral education target in university.

Secondly, we emphasize the pertinence of the construction. Because the needs of every student talent development are different, so we should design different moral education target in university for different students. According to different age groups, different knowledge abilities and different moral quality levels of students, we should design the targeted goal of moral education and adopt appropriate teaching tactics to ensure that moral education target and moral educator is harmonious and unified in moral education activities which is a major means to realizing the goal of moral education.

Thirdly, we emphasize the operability of the construction. It means that we should be based on the nature of the school, the facilities of the school, the ideological actual situation of students' and the needs of students' to build a harmonious moral education target in university. The construction of moral education target is the prerequisite for the implementation of moral education activities, the implementation of the activities of moral education is an important way for cultivating students to be a useful person. Only by carrying out moral education activities smoothly, we can make the students improve their moral level and ideological quality. The aim of emphasizing the operability of the construction is to improve the effectiveness of moral education activities, approach to moral education target.
The principles of building harmonious moral education target in university are a set of relatively complete system; it runs through all process of building harmonious moral education target. We must insist on correct guidance, reflect the students' subjectivity, establish the regularity, hierarchy and systematicness of building of moral education target, truly achieve the goal of moral education target harmony, get the harmonious moral education process and be to reveal the value of moral education.

\section{REFERENCES}

[1] Wansheng Zhan, "Theory of Harmonious Moral Education," Guangming Daily. Beijing, pp. 06, March 2006.

[2] E .Lenorad, "Jossem. Einstein's Theory of Education”, Physics and Engineering. Beijing, pp. 97, March 2005.

[3] K. Marx and F. Engels, Selections of K. Marx and F. Engels, vol. 3. Beijing: People's Publishing House, 1995, pp.254.

[4] Wansheng Zhan, Building a System of Moral Education.. Beijing: Science and Education Publishing House, 2001, pp.227.

[5] Enyun Liu, "Thoughts of Moral Education Function in Colleges and Building of Moral Education" Jiangsu Higher Education. Nanjing, pp. 35 , June 2002

[6] Xingfu Wu., “ Integrate the Principles of Constructing Higher Vocational Moral Education Target" Proceedings of the national academy of education administration, Taiyuan, pp. 155, February,2002

[7] Rong Yu, "Construction of the Harmonious Moral Mducation Target Standard System" Journal of Changchun university of technology, Changchun, pp. 54, March 2010. 